\section{Acute angle closure glaucoma: an evaluation of a protocol for acute treatment}

\begin{abstract}
Purpose To report the use of a protocol for the treatment of acute angle closure glaucoma (AACG) and document its effectiveness. Methods Following a clinical audit, a formal protocol for the treatment of AACG was introduced in our department. Three and a half years later, the records of 63 consecutive patients were reviewed. A descriptive analysis was performed.

Results At presentation the mean intraocular pressure (IOP) in the affected eye was $56 \mathrm{mmHg}$. The visual acuity was $6 / 60$ or worse in $68 \%$ of patients. The mean duration to achieve adequate IOP control was $3 \mathrm{~h}$ (range 1-7 h) and $44 \%$ of the patients achieved this without the use of osmotic diuretics. None of the fellow eyes developed AACG prior to peripheral iridotomy. No further medical or surgical treatment was needed in $44 \%$. Further topical treatment alone and surgical treatment were needed in $21 \%$ and $35 \%$ respectively. Following adequate IOP control, $76 \%$ had a visual acuity of $6 / 24$ or better. All who had a final visual acuity of $6 / 60$ or worse had significant non-glaucomatous pathology. At 6 months follow-up, $67 \%$ were treatment free and $65 \%$ had a normal optic disc.

Conclusions This study demonstrates that this treatment protocol provided comprehensive and explicit guidance on the emergency treatment of AACG. This resulted in rapid IOP control following presentation and eventual favourable outcome in most cases.
\end{abstract}

Key words Acute angle closure glaucoma, Treatment protocol

Acute angle closure glaucoma (AACG) is a common ophthalmic emergency, which requires early recognition followed by appropriate treatment to minimise visual loss. The urgent necessity to lower intraocular pressure is well known. This is normally achieved using medical therapy such as miotics, carbonic anhydrase inhibitors, beta blockers and osmotic diuretics. This is followed by definitive treatment such as a Nd:YAG laser peripheral
YEE FONG CHOONG, SAMEERA IRFAN, MITCHEL J. MENAGE

iridotomy (PI) or in some cases filtration surgery. It is a condition commonly presenting out of normal working hours and often is initially managed by the least experienced members of the medical team.

There was a perceived variability in emergency treatment of AACG in our department and an audit of patients presenting prior to 1994 was carried out. This confirmed a rather diverse selection of drug regimes not all of which were deemed optimal. Consequently a standard protocol for acute management of the condition was drafted and posted in the department and circulated to medical and nursing staff. The protocol was a modified version of that proposed by Kramer and Ritch and recently expanded by Ritch et al. ${ }^{1}$ The protocol was well received by junior staff who were only too pleased to receive explicit guidance on treatment.

Another audit exercise was carried out in late 1997 to evaluate the treatment protocol. The purpose of this paper is to report the departmental use of a protocol for this common condition and document its effectiveness.

\section{Patients and methods}

All patients attending The General Infirmary at Leeds presenting with AACG between September 1994 and February 1997 were included. The diagnosis was made clinically on the basis of a history of pain and visual loss, and the finding of raised intraocular pressure (IOP) as measured with Goldmann applanation tonometer, corneal oedema, a fixed mid-dilated pupil and a closed iridocorneal angle. The assessment of iridocorneal angle was made either by gonioscopy or by direct observation of iridocorneal touch on the slit-lamp. All the patients were admitted and were immediately commenced on treatment as recommended by the protocol (Table 1).

We divided the protocol into three stages. Stage 1 consisted of the immediate treatment on making the diagnosis. Acetazolamide (Diamox) $500 \mathrm{mg}$ was given intravenously and $500 \mathrm{mg}$ (not slow released) orally as stat doses. Topical beta blocker two times a day and topical steroid four times a day were started. The patient was
Y.F. Choong

S. Irfan

M.J. Menage

The Eye Department

Clarendon Wing

The General Infirmary

at Leeds

Belmont Grove

Leeds LS2 9NS, UK

Presented as a poster at the Annual Oxford Congress Meeting, 1998

Received: 23 November 1998 Accepted in revised form: 4 June 1999 


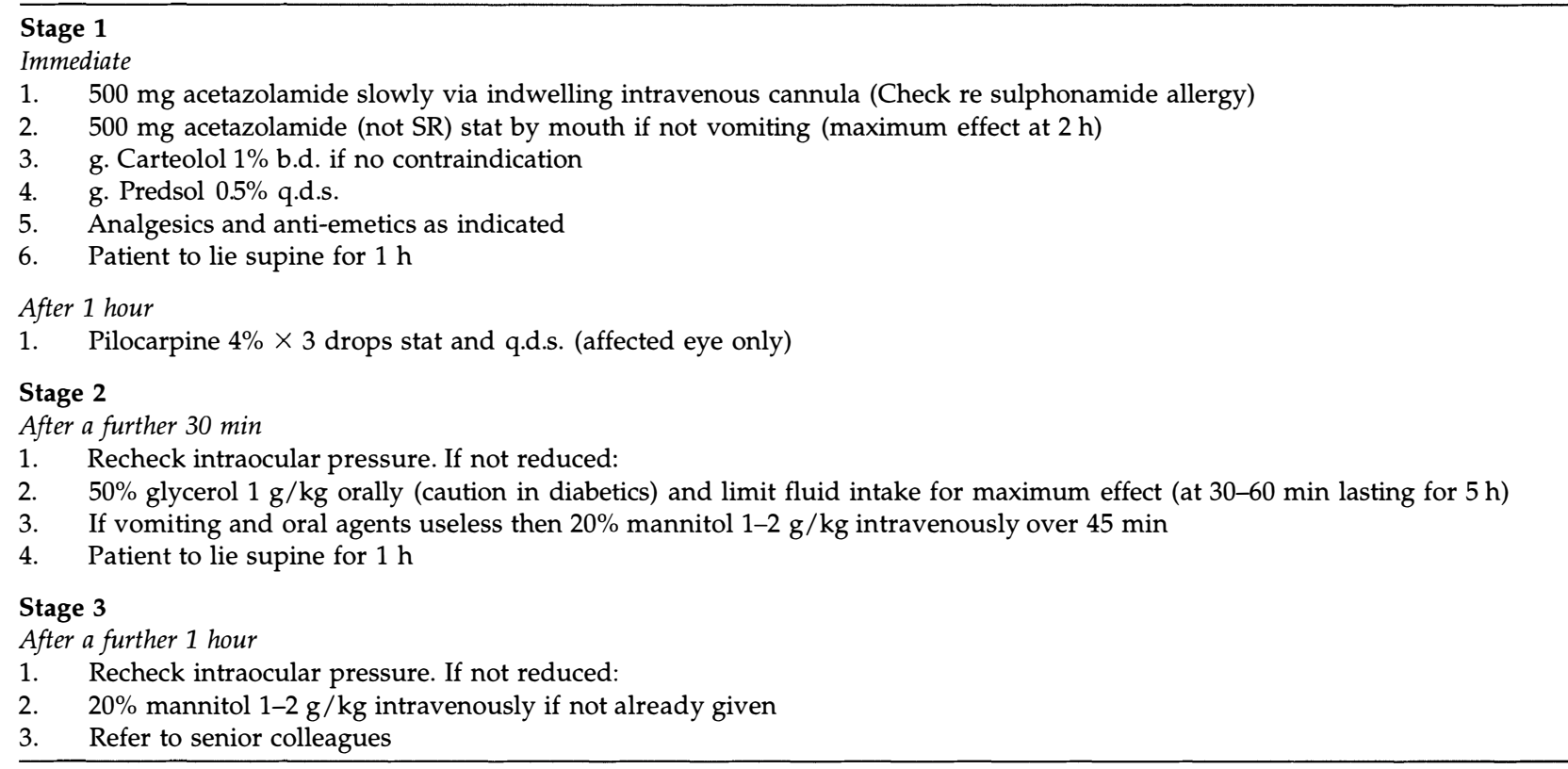

instructed to lie supine for an hour. Analgesics and antiemetics were given as indicated. After $1 \mathrm{~h}, 3$ drops of pilocarpine $4 \%$ were given as a stat dose in the affected eye only and this was repeated four times a day. We do not treat the fellow eye with topical treatment. The IOP was rechecked $30 \mathrm{~min}$ later, and if it was not satisfactorily reduced the patient was commenced on stage 2 of the treatment. Satisfactory IOP was defined as less than $35 \mathrm{mmHg}$ or a reduction of greater than $25 \%$ of the presenting IOP. In stage 2 of the treatment, $50 \%$ glycerol $1 \mathrm{~g} / \mathrm{kg}$ orally was given. This was substituted with $20 \%$ mannitol $1-2 \mathrm{~g} / \mathrm{kg}$ intravenously over $45 \mathrm{~min}$ if the patient was vomiting or unable to tolerate oral glycerol. The patient was to remain supine for another hour. After this the IOP was rechecked and, if it was not satisfactorily reduced, $20 \%$ mannitol $1-2 \mathrm{~g} / \mathrm{kg}$ was given intravenously if not already given or, if already given, was repeated. After the acute attack was adequately controlled, bilateral Nd:YAG laser peripheral iridotomy was performed using a Topaz Class IV Nd:YAG laser and Abraham contact lens. All antiglaucoma medication was stopped. If the pressure was not adequately controlled, antiglaucoma medication was recommenced. Surgical treatment followed if the IOP remained uncontrolled. The patients were seen in the outpatient department following discharge and had a minimum follow-up of 6 months.

Descriptive statistical analysis was performed with attention focused on the stages of treatment needed, the time required to achieve satisfactory IOP control, and the need for further medical and surgical therapy to achieve IOP control.

The patients were further divided into two groups: group A required no further treatment following PI, while group $B$ required anti-glaucoma medication and/or surgical treatment to achieve satisfactory IOP control. Thus group A represents patients with adequate angle function whereas group $B$ represents patients with angles which were functionally compromised. The mean duration of symptoms prior to presentation, a history of subacute attacks and the appearance of the optic disc were recorded. The assessment of the pathological state of the optic disc was made by the examining clinician on the basis of clinical judgement and comparison with the unaffected eye. Inevitably we would expect some inter/intra-observer error in grading of the cup-disc ratio. Comparison between the two groups was by Mann-Whitney $U$-test and chi-squared test.

\section{Results}

Sixty-three patients were seen over the duration of the study. All had AACG affecting only one eye. There were 49 women $(78 \%)$ and 14 men $(22 \%)$. One patient was Asian and the rest were Caucasians. Right eyes were involved in $36(57 \%)$ patients. The mean age of the patients was 72 years (range $35-100$ years). The duration of symptoms varied between 1 and 14 days (mean 2.5 days, median 1 day). The presenting visual acuity was $6 /$ 60 or worse in $43(68 \%)$ patients. Two patients were too confused to have their visual acuities measured. The mean presenting IOP in the affected eye was $56 \mathrm{mmHg}$ (median $54 \mathrm{mmHg}$, range $34-78 \mathrm{Hg}$ ). In the unaffected eye, the mean IOP was $17.5 \mathrm{mmHg}$ (median $17 \mathrm{mmHg}$, range $12-34 \mathrm{mmHg}$ ). A family history of glaucoma was

Table 2. Acute angle closure glaucoma: patient details

\begin{tabular}{lc}
\hline Number $(n)$ & 63 \\
Mean age (years) & 72 (range 35-100) \\
Sex & \\
$\quad$ Female & $49(78 \%)$ \\
$\quad$ Male & $14(22 \%)$ \\
Eye & \\
$\quad$ Right & $36(56 \%)$ \\
$\quad$ Left & $27(44 \%)$ \\
Mean presenting IOP (mmHg) & $56($ range 34-78) \\
Family history of glaucoma & $6(9 \%)$ \\
\hline
\end{tabular}


Table 3. Acute angle closure glaucoma: precipitating events

\begin{tabular}{lr}
\hline Iatrogenic pupillary dilatation & 5 \\
Amitriptyline usage & 2 \\
Intumescent cataract & 4 \\
Anterior uveitis & 1 \\
Total & 12 \\
\hline
\end{tabular}

found in $6(10 \%)$ patients (Table 2). Precipitating events were identified in $12(19 \%)$ patients (Table 3). These included iatrogenic pupillary dilatation, amitriptyline use for depression, intumescent cataracts and anterior uveitis. We were unable to determine from the medical notes whether any patients had plateau iris.

The treatment protocol was followed in all cases. Stage 1 of the treatment protocol was sufficient to achieve satisfactory IOP in $28(44 \%)$ patients. Of these patients, 3 had IOPs reduced to between 35 and $41 \mathrm{mmHg}$ but had achieved a greater than $25 \%$ reduction in IOP. Stages 1 and 2 were needed in $32(51 \%)$ patients, i.e. 32 of the 35 (91\%) remaining patients needed a single dose of osmotic diuretics. All three stages were needed in only $3(5 \%)$ patients (Table 4). The mean time required to achieve satisfactory IOP was $3 \mathrm{~h}$ (median $3 \mathrm{~h}$ ) following commencement of treatment. All patients responded satisfactorily within $7 \mathrm{~h}$. No patient developed any recognised side-effect related to the osmotic diuretics.

Nd:YAG PI was performed in $58(92 \%)$ of the affected eyes. Of the 5 affected eyes which did not have PI, 4 had intumescent cataracts which were subsequently removed during their admission. The remaining patient had a previous PI for chronic angle closure glaucoma and underwent combined cataract extraction and trabeculectomy. None of these 5 patients needed longterm anti-glaucoma treatment. PI was performed within 2 days in $88 \%(51 / 58)$ of the patients. Following PI the mean of the first IOP measurement was $24 \mathrm{mmHg}$ (median $23 \mathrm{mmHg}$ ) with 27 patients (43\%) having an IOP less than $22 \mathrm{mmHg}$ and 45 patients (71\%) an IOP less than $31 \mathrm{mmHg}$. No further treatment was needed in 28 (44\%) patients, $13(21 \%)$ had topical anti-glaucoma treatment alone, $12(19 \%)$ had trabeculectomy, $4(6 \%)$ had combined cataract extraction and trabeculectomy (phacotrabeculectomy), and $6(10 \%)$ had cataract extraction (Table 5).

The final visual acuity in the affected eye was better than or equal to $6 / 24$ in $48(76 \%)$ patients. The remaining 15 patients had final visual acuities of $6 / 60$ or worse in the affected eye and all of them had other significant ocular pathology. A branch retinal vein occlusion was found in 1 eye, 1 had a very pale atrophic disc, 2 had macular holes, 2 were amblyopic, 4 had very dense cataract, 2 had advanced macular degeneration and 3

Table 4. Acute angle closure glaucoma: treatment stages

\begin{tabular}{lrr}
\hline & $n$ & $\%$ \\
\hline Stage 1 & 28 & 44 \\
Stages 1 and 2 & 32 & 51 \\
Stages 1,2 and 3 & 3 & 5 \\
Total & 63 & 100 \\
\hline
\end{tabular}

Table 5. Acute angle closure glaucoma: further treatment required

\begin{tabular}{lrr}
\hline & $n$ & $\%$ \\
\hline None & 28 & 44 \\
Topical anti-glaucoma treatment alone & 13 & 21 \\
Trabeculectomy & 12 & 19 \\
Phacotrabeculectomy & 4 & 6 \\
Cataract extraction & 6 & 10 \\
Total & 63 & 100 \\
\hline
\end{tabular}

had a combination of cataract and macular degeneration. The optic discs were normal in 41 (65\%) patients, pathologically cupped in $16(25 \%)$, atrophic in $4(6 \%)$, ischaemic in $1(2 \%)$ and unknown in $1(2 \%)$ (Table 6). At 6 months follow-up, $42(67 \%)$ patients were treatmentfree and 18 (29\%) were using topical anti-glaucoma treatment alone. Three patients were lost to follow-up, of whom 2 died from unrelated medical conditions within 6 months of presentation to our department.

The mean duration of symptoms prior to presentation in group A and group B was 2.0 days (median = 1 day) and 2.9 days (median $=1$ day) respectively. The percentage of patients with a subacute history in group A and $B$ was $43 \%(12 / 28)$ and $57 \%(20 / 35)$ respectively. There was no significant difference in the duration of symptoms prior to presentation $(p>0.05)$ or in a history of subacute attacks $\left(\chi^{2}=1.27\right.$, d.f. $\left.=1, p=0.26\right)$ between the two groups,. In group $\mathrm{A}$, the discs were cupped or atrophic in 5 of $28(18 \%)$ patients, whereas in group B chronic disc damage was found in 15 of $34(44 \%)$ patients. We have included the patient with the acute ischaemic disc in the normal group as it was assumed not to have been chronically damaged before presentation. There were significantly more chronically damaged discs in group B $\left(\chi^{2}=4.85\right.$, d.f. $\left.=1, p=0.028\right)$.

\section{Discussion}

There are very few recent data published on the initial treatment of AACG and its efficacy. Although the management of AACG is discussed in the major textbooks there is considerable variation in the details of treatment between and even within eye departments. This observation in our own department prompted the development of a protocol. In this study we showed that our protocol was reliable in terminating the acute attack in the vast majority of cases. Only $5 \%$ of patients entered stage 3 of the protocol to achieved satisfactory IOP control and all our patients responded satisfactorily within $7 \mathrm{~h}$ (mean $3 \mathrm{~h}$ ) following commencement of treatment. Acetazolamide $500 \mathrm{mg}$ intravenously and

Table 6. Acute angle closure glaucoma: optic disc appearance

\begin{tabular}{lccrr}
\hline & Group A & Group B & \multicolumn{2}{c}{ Total } \\
\cline { 3 - 5 } & $n$ & $n$ & $n$ & $\%$ \\
\hline Normal & 23 & 18 & 41 & 65 \\
Cupped & 3 & 13 & 16 & 25 \\
Atrophic & 2 & 2 & 4 & 6 \\
Ischaemic & 0 & 1 & 1 & 2 \\
Unknown & 0 & 1 & 1 & 2 \\
Total & 28 & 35 & 63 & 100 \\
\hline
\end{tabular}


$500 \mathrm{mg}$ orally were given as stat doses to the patient together with a topical beta blocker and topical steroid as soon as the diagnosis was made. Acetazolamide is effective in lowering IOP and opening the anterior chamber angle in AACG. ${ }^{2}$ Beta blocker is used as an additive to acetazolamide in lowering IOP and topical steroid acts as an anti-inflammatory agent. Pilocarpine was given $1 \mathrm{~h}$ after initial reduction of IOP because of iris sphincter ischaemia and paralysis at high IOP.

Pilocarpine induces pupillary constriction which leads to opening of the narrow angle and thus facilitates aqueous outflow, but it also causes shallowing of the anterior chamber by increasing axial lens thickness and inducing anterior lens movement. ${ }^{3-5}$ The latter actions may worsen the situation in what is known as the paradoxical reaction to pilocarpine. ${ }^{6,7}$

The management of the fellow eye in AACG is somewhat controversial. While Nd:YAG peripheral iridotomy has been established as a prophylactic treatment of choice, the role of pilocarpine as prophylactic treatment prior to planned iridotomy is uncertain. ${ }^{8,9}$ A survey of members of the American Glaucoma Society showed that pilocarpine alone was used as the treatment of the fellow eye in cases when iridotomy was temporarily deferred in more than half of the respondents whereas close observation was the choice of a third. ${ }^{10}$ However, we did not treat the fellow eye prophylactically with pilocarpine prior to iridotomy for fear of inducing angle closure. PI was carried out within a short period in the majority of our patients. In this study none of the fellow eyes developed AACG prior to PI.

Oral glycerol and intravenous mannitol were our osmotic diuretics of choice. All osmotic diuretics must be administered with care as they can cause serious complications, especially in patients with cardiovascular and renal disease. We did not commence osmotic diuretics on presentation as a significant number of angle closure attacks can be aborted without using these potentially harmful drugs. This view is supported by this study, as $44 \%$ of the patients did not need any osmotic diuretic to achieve satisfactory IOP. Osmotic diuretics were effective in lowering IOP in cases which were resistant to the initial treatment. We did not encounter any serious complications from osmotic diuretics in this study.

Following iridotomy, more than two-fifths (44\%) of the patients did not require any further treatment. This compared favourably with $36.5 \%$ in a series reported by Saunders. ${ }^{11}$ One fifth required topical anti-glaucoma treatment alone and the remaining patients required surgical treatment. At 6 months follow-up, $29 \%$ of our patients needed anti-glaucoma medication compared with $39 \%$ in the series by Saunders. ${ }^{11}$ Including those who had undergone surgery, two-thirds of our patients were treatment free.

Ideally we would have liked to report the angle status after iridotomy in the patients. This is likely to have some bearing on the eventual success of treatment and requirement for further medical or surgical therapy. A persistently occluded angle despite reduction in IOP with medical treatment and iridotomy can cause IOP increase later. Regrettably in a study such as this with data collection from clinical notes this proved too unreliable because of variability in interpretation of angle status, ambiguous recording and missing data.

After 6 months of follow-up two-thirds of the patients had a normal disc and all those with significant loss of visual acuity (6/60 or worse) had non-glaucomatous pathology. This indicates that prompt treatment of AACG usually results in a favourable visual outcome.

Some previous studies have suggested that the duration of symptoms prior to presentation and a subacute history of AACG can be helpful in distinguishing between patients who require further medical or surgical treatment and those who do not. ${ }^{11,12}$ We did not find this distinction. We noted that patients in group B (those requiring further treatment) had significantly more chronic disc damage than group A (those that did not). This appears to have some predictive value for continued therapy after resolution of the AACG. The implication is that there is an underlying chronic glaucoma with an acute episode. However, it must be realised that this is not unexpected as patients with pathologically cupped discs will be more likely to receive further anti-hypertensive treatment than those with healthy discs.

In summary, this study demonstrates that our treatment protocol provided comprehensive and explicit guidance on the treatment of AACG. This resulted in rapid IOP control following presentation and eventual favourable outcome in most cases.

\section{References}

1. Ritch R, Shields MB, Krupin T. The glaucomas, 2nd ed., vol. 3, St Louis: Mosby-Year Book, 1996:1521.

2. Lowe RF. Acute angle closure glaucoma: acetazolamide therapy. Austral J Ophthalmol 1973;1:24.

3. Abramson DH, Chang S, Coleman DJ, et al. Pilocarpineinduced lens changes: an ultrasonic biometric evaluation of dose response. Arch Ophthalmol 1974;92:464.

4. Abramson DH, Coleman DJ, Forbes M, Franzen LA. Pilocarpine: effect on the anterior chamber and lens thickness. Arch Ophthalmol 1972;87:615.

5. Wilkie J, Drance SM, Schulzer M. The effects of miotics on the anterior chamber depth. Am J Ophthalmol 1969;68:78.

6. Bleiman B, Schawrtz AL. Paradoxical response to pilocarpine. Arch Ophthalmol 1979;97:1305.

7. Ritch R. The pilocarpine paradox [editorial]. J Glaucoma 1996;5:225.

8. Moster MR, Schwartz LW, Spaeth GL, et al. Laser iridotomy: a controlled study comparing argon and neodymium:YAG. Ophthalmology 1986;93:2024.

9. Rivera AH, Brown RH, Anderson DR. Laser iridotomy vs surgical iridectomy: have the indications changed? Arch Ophthalmol 1985;103:1350.

10. Davidorf JM, Bajer ND, Derick R. Treatment of the fellow eye in acute angle-closure glaucoma: a case report and survey of members of the American Glaucoma Society. J Glaucoma 1996;5:228.

11. Saunders DC. Acute closed-angle glaucoma and Nd-YAG laser iridotomy: Br J Ophthalmol 1990;74:523.

12. Hass J, Scheie H. Peripheral iridectomy in narrow angle glaucoma. Ophthalmology 1957;55:59. 